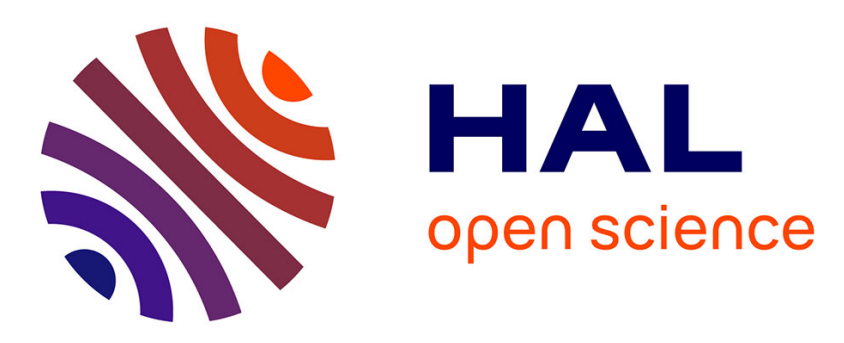

\title{
Évolution de l'exposition au bruit chez des salariés en France
}

\author{
Jean-Baptiste Pelletan, Emmanuel Fort, Laurène Delabre, Amélie \\ Massardier-Pilonchery, Corinne Pilorget
}

\section{- To cite this version:}

Jean-Baptiste Pelletan, Emmanuel Fort, Laurène Delabre, Amélie Massardier-Pilonchery, Corinne Pilorget. Évolution de l'exposition au bruit chez des salariés en France. 35e congrès national de médecine et santé au travail, Jun 2018, MARSEILLE, France. pp. 413-414, 10.1016/j.admp.2018.03.459 . hal02265979

\section{HAL Id: hal-02265979 \\ https://hal.science/hal-02265979}

Submitted on 13 Aug 2019

HAL is a multi-disciplinary open access archive for the deposit and dissemination of scientific research documents, whether they are published or not. The documents may come from teaching and research institutions in France or abroad, or from public or private research centers.
L'archive ouverte pluridisciplinaire HAL, est destinée au dépôt et à la diffusion de documents scientifiques de niveau recherche, publiés ou non, émanant des établissements d'enseignement et de recherche français ou étrangers, des laboratoires publics ou privés. 
En France, la surdité professionnelle est la $3^{\mathrm{e}}$ cause de maladie professionnelle reconnue par les régimes général et agricole de la sécurité sociale.

Entre 1968 et 2000, l'institut universitaire de médecine du travail de Lyon a mené des campagnes d'examens audiométriques dans des entreprises de la région Rhône-Alpes afin d'évaluer l'impact des nuisances sonores sur les travailleurs. L'exploitation de ces données permet d'appréhender l'évolution de l'exposition professionnelle au bruit lésionnel sur cette période. Ce travail s’inscrit dans le cadre du programme Matgéné, il a pour finalité d'élaborer une matrice emploi exposition au bruit.

\section{Matériel et méthodes}

La base de données utilisée comporte 49600 examens audiométriques. Les informations sur les emplois ont été codées selon la PCS 2003 (profession et catégorie socioprofessionnelle) et la NAF 2008 (nomenclature des activités française).

Des critères de sélection sur l'âge, l'ancienneté dans l'emploi et l'absence de pathologies de l'oreille ont été retenus pour exploiter ces audiométries, ce qui a permis l'analyse de 8895 audiogrammes. Une perte auditive moyenne standard (PAM standard) a été estimée de manière collective, à partir des PAM calculées pour chaque salarié appartenant à un même groupe professionnel. Le niveau d'exposition sonore théorique du groupe Leq, exprimé en $\mathrm{dB}(\mathrm{A})$ est déterminé par la relation suivante $:$ Leq $=(\mathrm{PAM}$ standard $+43,3) / 0,688$.

Les niveaux sonores moyens et leurs intervalles de confiance à $95 \%$ ont été calculés selon le sexe, la profession et le secteur d'activité et déclinés sur 4 périodes distinctes : 1968-1969, 1970-1979, 1980-1989 et 1990-2000.

\section{Résultats}

La population est constituée majoritairement d'hommes (86,1 \%), l'âge moyen est de 38,9 ans $($ std $=9,2)$ et l'ancienneté moyenne de 14,4 ans (std = 7,9).

De manière globale, on constate une diminution significative de l'exposition sonore dans cette population : de 114,1 dB(a) (IC95 \% = [112,7-115,5]) à 86,9 dB(a) (IC95 \% = [85,6-88,2]) entre 1968 et 2000 . Cette tendance se confirme pour tous les secteurs d'activités exceptés dans l'industrie du papier et du carton et dans la cokéfaction et raffinage pour lesquels le niveau de bruit reste stable.

\section{Conclusion}

L'étude de mesures audiométriques réalisées sur une longue période historique a donc permis de documenter l'évolution de l'exposition professionnelle au bruit au sein d'un groupe professionnel. La baisse constatée des niveaux d'exposition sonore au cours du temps dans cette population est à mettre en parallèle avec les actions engagées par le droit français et européen depuis 1970 visant l'amélioration des conditions de travail. 